\title{
Closed loop identification of a piezoelectrically controlled radial gas bearing: Theory and experiment
}

Sekunda, André Krabdrup; Niemann, Hans Henrik; Poulsen, Niels Kjølstad; Santos, Ilmar Ferreira

Published in:

Proceedings of the Institution of Mechanical Engineers, Part I: Journal of Systems and Control Engineering

Link to article, DOI:

$10.1177 / 0959651818769230$

Publication date:

2018

Document Version

Peer reviewed version

Link back to DTU Orbit

Citation (APA):

Sekunda, A. K., Niemann, H. H., Poulsen, N. K., \& Santos, I. F. (2018). Closed loop identification of a piezoelectrically controlled radial gas bearing: Theory and experiment. Proceedings of the Institution of Mechanical Engineers, Part I: Journal of Systems and Control Engineering, 232(7), [095965181876923]. https://doi.org/10.1177/0959651818769230

\section{General rights}

Copyright and moral rights for the publications made accessible in the public portal are retained by the authors and/or other copyright owners and it is a condition of accessing publications that users recognise and abide by the legal requirements associated with these rights.

- Users may download and print one copy of any publication from the public portal for the purpose of private study or research.

- You may not further distribute the material or use it for any profit-making activity or commercial gain

- You may freely distribute the URL identifying the publication in the public portal 


\title{
Closed Loop \\ Identification of a Piezo Electrically-Controlled Radial Gas Bearing - Theory \& Experiment
}

Journal Title $X X(X): 2-20$

(C)The Author(s) 0000

Reprints and permission:

sagepub.co.uk/journalsPermissions.nav

DOI: $10.1177 /$ ToBeAssigned

www.sagepub.com/

(S)AGE

\section{André Sekunda ${ }^{1}$, Henrik Niemann ${ }^{1}$, Niels Kjølstad Poulsen ${ }^{2}$ and IImar Santos ${ }^{3}$}

\begin{abstract}
Gas bearing systems have extremely small damping properties. Feedback control is thus employed to increase the damping of gas bearings. Such a feedback loop correlates the input with the measurement noise which in turn makes the assumptions for direct identification invalid. The originality of this paper lies in the investigation of the impact of using different identification methods to identify a rotor-bearing systems dynamic model when a feedback loop is active. Two different identification methods are employed. The first method is open loop Prediction Error Method (PEM) while the other method is the modified Hansen scheme. Identification based on the modified Hansen scheme is conducted by identifying the Youla deviation system using subspace identification. Identification of the Youla deviation system is based on the Youla-Jabr-Bongiorno-Kucera parametrisation of plant and controller. By using the modified Hansen scheme, identification based on standard subspace identification methods can be used to identify the Youla deviation system of the gas bearing. This procedure ensures the input to the Youla deviation system and the noise are uncorrelated even though the system is subject to feedback control. The effect of identifying the Youla deviation system compared to direct subspace identification of the gas bearing is further investigated through a simulation example. Experiments are conducted on the piezoelectrically-controlled radial gas bearing. A dynamic model is identified using the modified Hansen scheme as well as using PEM identification. The resulting models are compared for different imperfect nominal models, to examine under which conditions each method should be used.
\end{abstract}

\section{Keywords}

Experiment, Gas Bearing, Closed-loop Identification, Coprime Factorisation, Subspace Identification 


\section{Introduction}

Active gas bearings are an interesting alternative to more commonly used bearings such as ball and magnetic bearings. Compared to ball bearings, active gas bearings have extremely low friction. At the same time active gas bearings are open loop stable for appropriately low rotational speeds, unlike magnetic bearings, which are always open loop unstable. The active gas film itself delivers low damping. Because of the low damping the rotor system may become unstable due to self-excited rotor whirling. Control is therefore still required although it is less limited than for unstable systems. Designing models of gas bearings has however been shown to be less straightforward, compared to the frictionless alternative of magnetic bearings. A successful mathematchical model for active gas bearings was first introduced in ${ }^{1}$ with extensions to the model presented in ${ }^{2-4}$. Recently a low order model able to describe the dynamics of the active gas bearing was presented in ${ }^{5}$. Such low order models greatly simplify the control design phase.

Identification of gas bearings have been a subject of some interest recently ${ }^{6,7}$. It has been shown possible to identify appropriately low order models that mimic the behaviour of the gas bearing in a satisfying manner when no feedback loop is active. Usually the gas bearing will operate under conditions at which the damping is so small that feedback control is needed for safe operation. It is therefore of extreme relevance to be able to identify an appropriate model for the gas bearing under conditions where it is only possible to obtain identification data while the gas bearing is part of a closed loop system.

There are plenty of different methods for identifying closed loop systems. One of such methods transforms the closed loop identification problem of the plant into an open loop identification problem by using the Youla parameterization. Well known subspace identification methods are in this paper used for identifying the Youla deviation system. It has become increasingly popular to use subspace identification methods due to their natural connection to multiple-input and multiple-output state space models ${ }^{8}$. Some of the most well known subspace identification methods are the Numerical algorithm for Subspace IDentification (N4SID) proposed in ${ }^{9}$ and the Multivariable OutputError State-sPace (MOESP) proposed in ${ }^{10}$. Both methods, as with most subspace identification methods, assume the noise and input to be uncorrelated, hence the methods are based on assumptions that are only valid for open loop identification. Some work has been done on extending the subspace identification methods to identification of closed loop systems. Several different methods for coping with feedback connection

\footnotetext{
${ }^{1}$ Technical University of Denmark, Department of Electrical Engineering, DK

${ }^{2}$ Technical University of Denmark, Department of Applied Mathematics and Computer Science, DK

${ }^{3}$ Technical University of Denmark, Department of Mechanical Engineering, DK

Corresponding author:

André Sekunda, Technical University of Denmark Department of Electrical Engineering, Elektrovej, Lyngby, DK.

Email: aksek@elektro.dtu.dk
}

Prepared using sagej.cls 
have been proposed ${ }^{8}$. However, often such methods show the same weakness of depending on being able to identify the noise signal imposed on the system.

One possible indirect identification method is subspace identification of the Youla deviation system. The Youla deviation system is used to reformulate the identification problem from a closed loop to an open loop identification problem using a coprime factorisation of the nominal plant model and the controller. This identification procedure is known as the Hansen scheme and was first introduced in ${ }^{11}$. The original version of the Hansen scheme used external excitation signals to indirectly excite the Youla deviation system. A modified version of the Hansen scheme, first introduced in ${ }^{12}$, is used instead in this paper which makes it possible to directly impose an excitation signal onto the Youla deviation system. A gas bearing easily becomes an unstable system when the rotational speed is increased. However the Youla deviation system identified by the modified Hansen scheme will always be stable. The open loop identification technique is thus applicable where direct open loop identification of the gas bearing is not. The modified Hansen scheme however, has the drawback of loss of physical understanding of the system.

The key contribution of this paper is to offer insight into when it can be advantageous to identify an active gas bearing using the modified Hansen scheme instead of the more traditional Prediction Error Method identification. This paper thus focuses on comparing known identification techniques for identification of a gas bearing when being part of a closed loop system. The results are gathered by a combination of simulations and experiments conducted on a laboratory installation.

The paper is structured as follows: In the following section the model of the gas bearing is introduced; In the third section some preliminary theory is introduced in order to let the paper stand alone; In the fourth section the three methods used for identification of the gas bearing are discussed; In the fifth section the identification procedure is given and the quality of the nominal model is discussed; In sixth section the experimental methods are presented and the resulting model identified using each of the 3 different identification methods are shown. Finally, in the last section, a discussion of the results and future possible improvements are given.

\section{Nomenclature \\ - LFT \\ - LQR \\ - PEM \\ - MIMO \\ - MOESP \\ Linear Fractional Transformation \\ Linear-Quadratic Regulator \\ Predicition Error Method \\ Multiple Input Multiple Output \\ - N4SID \\ Multivariable Output-Error State-Space \\ Numerical algorithm for Subspace Identification}

\section{Gas Bearing System}

The gas bearing test rig used in this paper has already been subject to different identification methods, with the focus on its physical behavior in ${ }^{2,13,14}$ and on the 
system dynamics in ${ }^{5-7}$. These methods however, have all been both conducted and verified under open loop conditions. All identification in this paper are based on the gas bearing being subject to feedback control. The nominal model is based on the model structure introduced in ${ }^{5}$ which showed it possible to model the vertical and horizontal position of a disc attached to a flexible shaft. The shaft is held in position by the gas bearing, using gas injected into the bearing in both the horizontal and vertical directions using a 6'th order model. A picture of the test rig is presented on Fig. 1a.

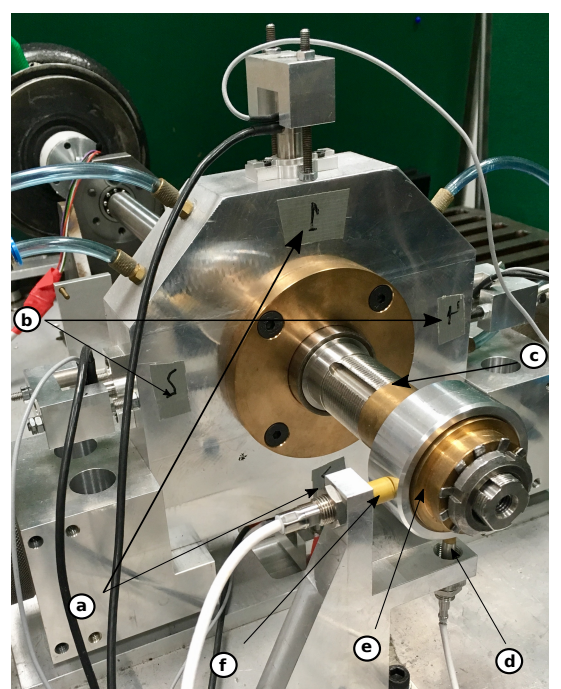

(a) Picture of the experimental test rig used for conducting active fault detection. The different parts of the test rig is as follows: a; vertical piezo actuator, b; horizontal piezo actuators, c; flexible shaft, d; vertical displacement sensor, e; disc and $\mathbf{f}$ horizontal displacement sensor.

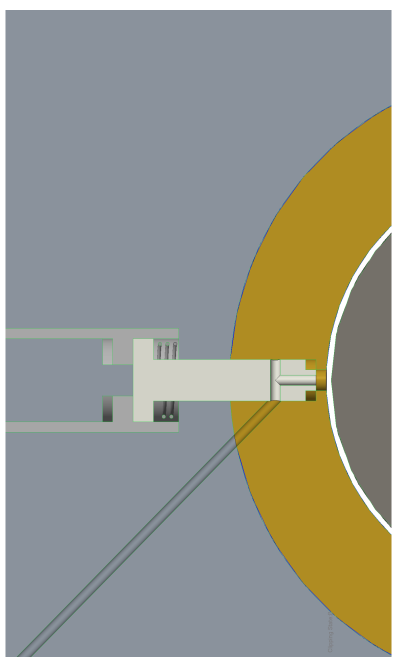

(b) Cross section illustration of the actuator. The further out the pin is moved the more air is able to flow in which increase the pressure. The position of the shaft is thus controlled by changing the flow of air through the inlet valve.

\section{Figure 1}

A illustration of the gas bearing actuators is shown on Figure 1b. The air is going into the bearing through the small pipe, a piezo electric actuator is attached which controls how much air is able to get into the bearing and thus controls the preasure inside the bearing.

The model of the gas bearing is based on the mass-springer-damper-model in Eq. (1) for the non rotating case.

$$
\ddot{\mathbf{I}}-\mathbf{D} \mathbf{i}-\mathbf{K} \mathbf{l}=\mathbf{B m}
$$


Here $\mathbf{l}$ is a vector with the position in the horizontal and vertical direction and $\mathbf{m}$ is the state of the actuator dynamics. $\mathbf{K}$ is the specific stiffness matrix given in $\left[\frac{N}{k g \mu m}\right], \mathbf{D}$ is the specific damping matrix in $\left[\frac{N s}{k g \mu m}\right]$, and $\mathbf{B}$ is the actuator gain matrix in $\left[\frac{N}{k g V}\right]$. Using Eq. (1) the state vector is defined in Eq. (2).

$$
\mathbf{x}=\left[\begin{array}{l}
\mathbf{l} \\
\mathbf{i} \\
\mathbf{m}
\end{array}\right]
$$

The structure of the state space model is given in Eq. (3) to Eq. (6) where each element is a $2 \times 2$ matrix

$$
\begin{aligned}
\mathbf{A} & =\left[\begin{array}{ccc}
0 & \mathbf{I} & 0 \\
\mathbf{K} & \mathbf{D} & \mathbf{B} \\
0 & 0 & -\mathbf{P}
\end{array}\right] \\
\mathbf{B} & =\left[\begin{array}{l}
0 \\
0 \\
\mathbf{P}
\end{array}\right] \\
\mathbf{C} & =\left[\begin{array}{lll}
\mathbf{I} & 0 & 0
\end{array}\right] \\
\mathbf{D} & =\left[\begin{array}{ll}
0
\end{array}\right]
\end{aligned}
$$

The matrix $\mathbf{P}$ is a diagonal matrix with each element $p_{j}$ defined by Eq. (7).

$$
h_{j}(s)=\frac{p_{j}}{s+p_{j}} \quad j \in\{1,2\}
$$

$h_{j}$ is the first order low pass filter through which the system is actuated. The state space model is thus given in Eq. (8).

$$
G:=\left\{\begin{array}{ll}
\dot{x} & =\mathbf{A} x+\mathbf{B} u \\
y & =\mathbf{C} x+\mathbf{D} u
\end{array}\right\}
$$

In order to conduct closed loop identification of the system, the parameters of the model have been identified with the disc not rotating as part of an open loop scheme. The identified matrices are given in Eq. (9) to Eq. (12)

Prepared using sagej.cls 


$$
\begin{aligned}
D & =\left[\begin{array}{cc}
-224.9 & 3.97 \\
9.12 & -267.7
\end{array}\right] \\
K & =\left[\begin{array}{cc}
-1.869 \cdot 10^{6} & -8577 \\
-9510 & -1.737 \cdot 10^{6}
\end{array}\right] \\
B & =\left[\begin{array}{cc}
-6.126 \cdot 10^{6} & 3.154 \cdot 10^{5} \\
-1.571 \cdot 10^{5} & -4.516 \cdot 10^{6}
\end{array}\right] \\
P & =\left[\begin{array}{cc}
989 & 0 \\
0 & 942.5
\end{array}\right]
\end{aligned}
$$

\section{Theory}

The first part of this section is dedicated to presenting some definitions to the reader in order to make the rest of the paper stand alone. A nominal plant is given by $G(0)$ and a stabilizing controller is given by $K$. The coprime factorization of $G(0)$ and $K$ are given by:

$$
\begin{aligned}
G(0) & =N M^{-1}=\tilde{M}^{-1} \tilde{N}, \quad N, M, \tilde{N}, \tilde{M} \in R H_{\infty} \\
K & =U V^{-1}=\tilde{V}^{-1} \tilde{U}, \quad U, V, \tilde{U}, \tilde{V} \in R H_{\infty}
\end{aligned}
$$

Here $N$ and $M$ denote the right coprime factorisation of the nominal plant and $\tilde{N}$ and $\tilde{M}$ denote the left coprime factorisation of the nominal plant. Equivalently the right coprime factorisation of the controller is given by $V$ and $U$, and the left coprime factorisation is given by $\tilde{V}$ and $\tilde{U}$ such that the Bezout identity given in Eq. (15) is satisfied.

$$
\left[\begin{array}{ll}
I & 0 \\
0 & I
\end{array}\right]=\left[\begin{array}{ll}
M & U \\
N & V
\end{array}\right]\left[\begin{array}{cc}
\tilde{V} & -\tilde{U} \\
-\tilde{N} & \tilde{M}
\end{array}\right]=\left[\begin{array}{cc}
\tilde{V} & -\tilde{U} \\
-\tilde{N} & \tilde{M}
\end{array}\right]\left[\begin{array}{cc}
M & U \\
N & V
\end{array}\right]
$$

It will be assumed in this paper that $K$ is a stabilizing controller for both the nominal plant as well as for the real plant $G(S)$. This is the case if Eq. (15) is true. Here $S$ is the Youla deviation system describing the divergence between the nominal model and the real plant. With the controller and plant model factorisations defined in Eq. (13) and (14) the real plant is given by Eq. (16) using the right coprime factorisation and Eq. (17) using the left corpime factorisation.

$$
\begin{aligned}
& G(S)=(N+V S)(M+U S)^{-1}, S \in \mathcal{R} \mathcal{H}_{\infty} \\
& G(S)=(\tilde{M}+S \tilde{U})^{-1}(\tilde{N}+S \tilde{V}), S \in \mathcal{R} \mathcal{H}_{\infty}
\end{aligned}
$$


With a formulation of the true plant as in Eq. (17) only the Youla deviation $\operatorname{system}(S)$ is unknown and needs to be identified. $\mathrm{G}(\mathrm{S})$ from Eq. (16) can be formulated using a linear fractional transformation (LFT) given by:

$$
G(S)=\mathcal{F}_{u}\left(J_{G}, S\right)
$$

where:

$$
J_{G}=\left[\begin{array}{cc}
-M^{-1} U & M^{-1} \\
\tilde{M}^{-1} & G(0)
\end{array}\right]
$$

Here the input and output signals of $J_{G}$ is presented in Eq. (19).

$$
\left[\begin{array}{l}
\eta \\
y
\end{array}\right]=J_{G}\left[\begin{array}{l}
\epsilon \\
u
\end{array}\right]
$$

The closed-loop system using the system description in Eq. (18) is shown in Fig. 2, where $v_{1}$ and $v_{2}$ are two possible excitation signals and $n$ is the noise.

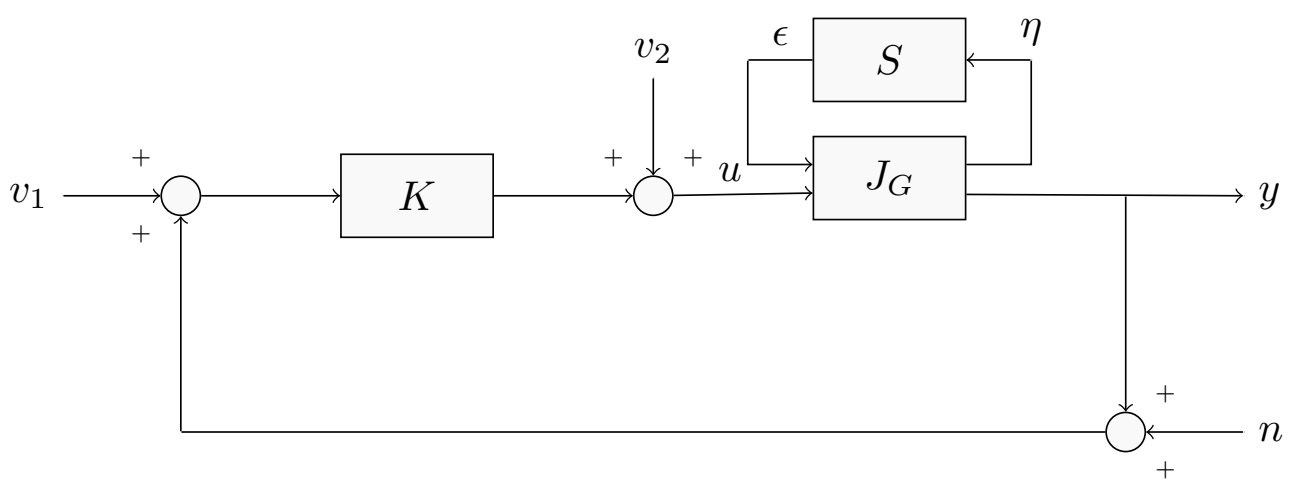

Figure 2. Closed loop system with the plant represented as a nominal part $\left(J_{G}\right)$ and the unknown Youla deviation system $S$.

Using Eq. (18) and the setup in Fig. 2, the vector introduced, $\epsilon$, is given in Eq. (20).

$$
\epsilon=S \eta
$$

Eq. (20) shows that the identification of $\mathrm{S}$ is straightforward using the signals $\eta$ and $\epsilon$. The two signals $\epsilon$ and $\eta$ in Eq. (20) are not directly accessible. However, it is shown

Prepared using sagej.cls 
that using the available signal vectors $v_{1}, v_{2}, y$ and $u$ it is possible to generate $\epsilon$ and $\eta$. It is therefore not possible to inject $\eta$ directly for the open loop identification of $\mathrm{S}^{11}$. Instead, including the Youla parametrization of all controllers in the closed-loop, it will be possible to have a direct access to $\epsilon$ and $\eta$ as shown further on. Let the Youla parametrization of all stabilizing controllers for the nominal system $G(0)$ be given by the following LFT description:

$$
K(Q)=\mathcal{F}_{l}\left(J_{K}, Q\right)
$$

where:

$$
J_{K}=\left[\begin{array}{cc}
K & \tilde{V}^{-1} \\
V^{-1} & -V^{-1} N
\end{array}\right]
$$

The input and output signals of $J_{K}$ are in Eq. (22).

$$
\left[\begin{array}{l}
u \\
\beta
\end{array}\right]=J_{K}\left[\begin{array}{l}
y \\
\alpha
\end{array}\right]
$$

Again it is possible to introduce two new signals in order to determine Q.

$$
\alpha=Q \beta
$$

The two new signals $\beta$ and $\alpha$ are part of the controller and can thus be directly measured and inserted. The relationship between the signals in the plant and controller can therefore give a significant advantage for direct identification of $S$.

The relationship between the signals $\eta, \epsilon, \beta$ and $\alpha$ can be calculated as a Redheffer star product as defined in ${ }^{15}$. The resulting relationship is shown in Eq. (24).

$$
\left[\begin{array}{l}
\alpha \\
\beta
\end{array}\right]=J_{G} \star J_{K}=\left[\begin{array}{ll}
1 & 0 \\
0 & 1
\end{array}\right]\left[\begin{array}{l}
\eta \\
\epsilon
\end{array}\right]
$$

Here $\star$ denotes the redheffer star product. The Youla deviation system is therefore possible to identify using the signals found in the controller, which is used for the closed loop identification. Based on the system description given on Figure 2 the Youla deviation system can thus be identified using Eq. (25).

$$
\beta=S \alpha+(\tilde{M}+S \tilde{U}) n
$$

Prepared using sagej.cls 
This ability to directly impose the excitation signal on the Youla deviation system $S$ is the advantage of the modified Hansen scheme compared to the original version. The modification is believed to make the identification more intuitive and was first introduced in ${ }^{12}$.

\section{Identification methods}

In all tests conducted the plant is subject to a feedback loop with an a priori designed controller. In order to conduct a proper comparison between the methods, all methods use data from the same experiment. Furthermore the noise $(n)$ has been found to be approximately white gaussian from an open loop dataset without any excitation.

\section{Identification using the modified Hansen Scheme}

It was argued in ${ }^{12}$ that the signals should be obtained directly from the controller by modifying it to the form shown in Fig. 3

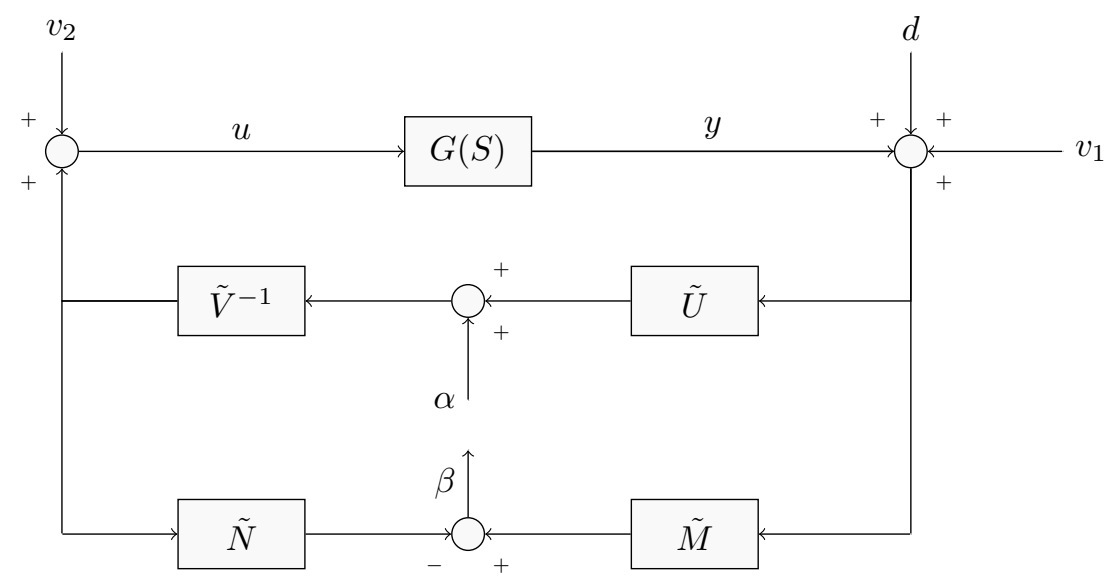

Figure 3. Representation of a YJKB parametrised controller for generation of signals for identification.

Using the signals $\alpha$ and $\beta$ it is possible to cast an identification problem as given in Eq. (25) by taking advantage of the relationship found in Eq. (24). Since $\alpha$ does not depend on the noise $n$ as shown in ${ }^{16}$ it is clear from Eq. (25) that the noise and excitation signal are not correlated. The system $S$ is identified using the subspace method N4SID for identification. The choice of using a subspace method for identification is two-fold. One of the great advantages of using subspace identification is the direct identification of MIMO state space models. The other important advantages of choosing a subspace identification method is that the methods are based on zero a priori knowledge. Unlike the true system $G(S)$ it is rather difficult to determine a proper initial structure of the Youla deviation system $S$. This comes from the fact that the nominal system is believed 
to be the best initial guess possible. All prior knowledge therefore points towards the Youla devitation system being 0 .

\section{Direct Grey Box Identification}

This identification method, as the name suggests, does not use any information regarding the feedback loop and the controller to determine the model. The method was presented in ${ }^{17}$ and is based on obtaining input and output data of the plant and determining the model. In this paper it is implemented as a PEM. The method uses the input-output data together with a nominal model as the information for parameter identification.

\section{Preliminary open loop identification}

Identification is conducted in two separate steps. First, a nominal model is generated to use as a basis for identification of the plant when part of a closed loop scheme. Such a model is obtained using data acquired from an open loop experiment conducted when the active gas bearing is not rotating. The identification results for the nominal model are based on the method described in ${ }^{7}$.

The identified nominal model is able to predict the displacement to an acceptable degree. The parameters identified through the experiment were presented in section . In order to give a quantitative measure of the quality of the different identification procedures used during the experiments, a goodness fit is calculated using Eq. (26) which is the normalised root mean square error.

$$
R^{2}=1-\frac{\|y-\hat{y}\|}{\|y-\bar{y}\|}
$$

Here $y$ is the measured output, $\bar{y}$ is the mean of the measured output and $\hat{y}$ is the estimated output using the model. Based on the nominal model, a controller is designed and a coprime factorisation is conducted. In the experiment only the left factorised form is used. In order to show the effect of the closed loop identification, the nominal model has been degraded in several different ways to examine the ability of the different identification procedures when the plant is part of a closed loop scheme. Lastly, a controller is been designed using the LQR function in MATLAB and implemented using the controller design presented in Fig. 3.

\section{Simulation Results}

It is in this section investigated which benefits there are from identifying the Youla deviation system. Identifying the Youla deviation system is compared to other more well known methods. It is decided to compare the method with grey box 
PEM identification and subspace identification. Normal subspace identification is independent of the nominal model. Grey box PEM identification on the other hand uses information from the nominal model.

\section{Inferior a priori knowledge of the system dynamics}

A definition of the inferior model from which the system has to be identified is needed to compare the identification methods. For the first test inferior knowledge about the system dynamics has been investigated. The system matrix of the initial model is defined in Eq. (27) where $A_{\text {model }}$ has inferior knowledge relative to the real system matrix $A$.

$$
A_{\text {model }}=(1-\theta) \cdot A
$$

Here $A$ is the system matrix of the system to be identified, $A_{\text {model }}$ is the system matrix of the nominal model and $\theta$ is a uniformly distributed random variable in the interval 0 to 1 . The nominal model thus contains a system matrix where all elements are smaller than for the real plant. A simulation has been conducted where 500 different initial models were constructed using Eq. (27). For each model a controller was designed that would stabilise the initial model and the real plant. Noise has been added as output noise and the system has been excited using a series of square waves. For each initial model an input-output set has been gathered and an identification has been conducted. In each case a second verification data set has been obtained where noise was omitted from the system. The identification results are shown on Fig. 4 for identification using PEM and subspace identification of the Youla deviation system.

It can be seen from Fig. 4 that for small deviations between the real system and the initial model both methods produce similar results. However as $\theta$ is increased the PEM identification starts to produce a lower $R^{2}$ fit.

With the methods compared it is concluded that identification of the Youla deviation system using subspace identification is resulting in higher $R^{2}$ fit when sufficient a priori knowledge of the system dynamics is lacking. Identifying the Youla deviation system instead of the plant directly, increases the complexity. The effect has therefore been examined when compared to direct subspace identification of the plant. Again 500 initial models were constructed using Eq. (27). Identification was conducted using subspace identification to identify the plant directly and to identify the Youla deviation system. A box plot of the resulting fit of the identified models is shown in Fig. 5.

It is seen from Fig. 5 that when the plant is directly identified, using a subspace identification method, the variance of the $R^{2}$ fit increases. Furthermore the mean of the $R^{2}$ fit is higher when identifying the Youla deviation system. Identifying the plant indirectly by identification of the Youla deviation system thus produces better results. Since both methods suffer from the loss of physical understanding of the models produced it is decided not to look further into direct subspace identification.

Prepared using sagej.cls 

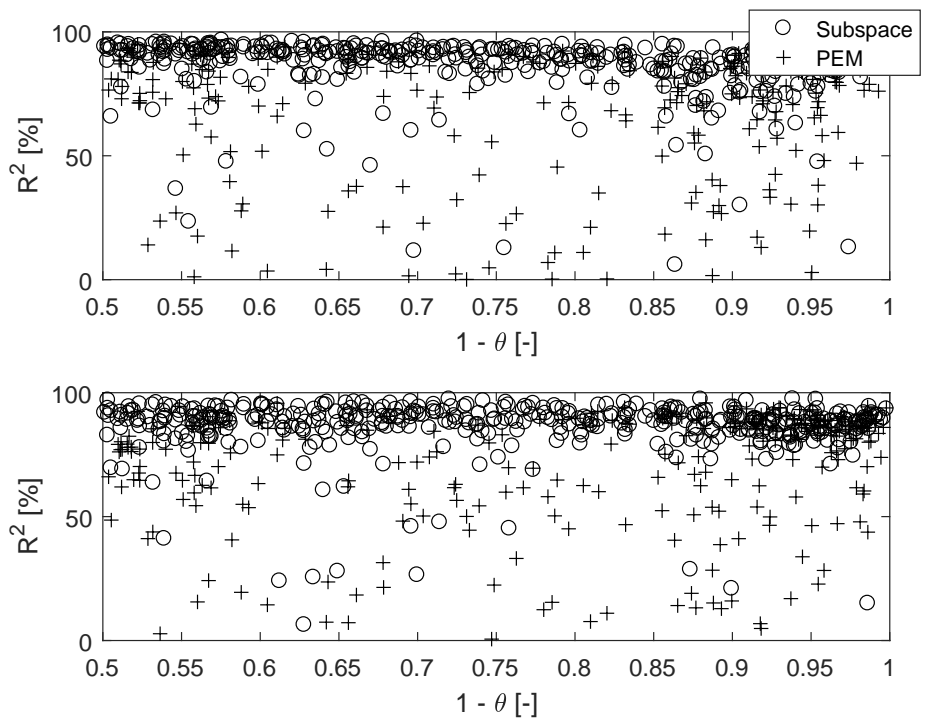

Figure 4. Plot of the $R^{2}$ fit in the horizontal direction (Top plot) and vertical direction (bottom plot) using the PEM for identification and subspace identification of the Youla system.

\section{Experiments \& Results}

With the controller designed and nominal model identified using the method described in $^{5}$, experiments have been conducted under 3 different conditions. The results have been summarised in Table 1 for all 3 experiments. In all experiments the same 2 identification methods have been applied; PEM using a fixed a priori model structure and identification of the Youla deviation system. The identification signals are found as Eq. (28) for direct identification of $S$. The system has been excited using the input signal denoted $\alpha$ on Fig. 3.

$$
\beta=\tilde{M} y-\tilde{N} u
$$

The Youla devitation system is the identified based on Eq. (25). The first experiment is conducted where the disc is non-rotating, and the nominal model has been degraded so that it is not able to mimic the plant dynamic in a satisfying manner. The degradation of the nominal model was achieved by multiplying all elements of the system matrix with 0.5 . The result of the identification is shown in Fig. 6 where the nominal model and the 2 identified models have been compared with a verification dataset.

It is shown in Fig. 6 that with a poor nominal model, identification of the Youla deviation system is able to recover the system dynamics. It is also noticed that the 

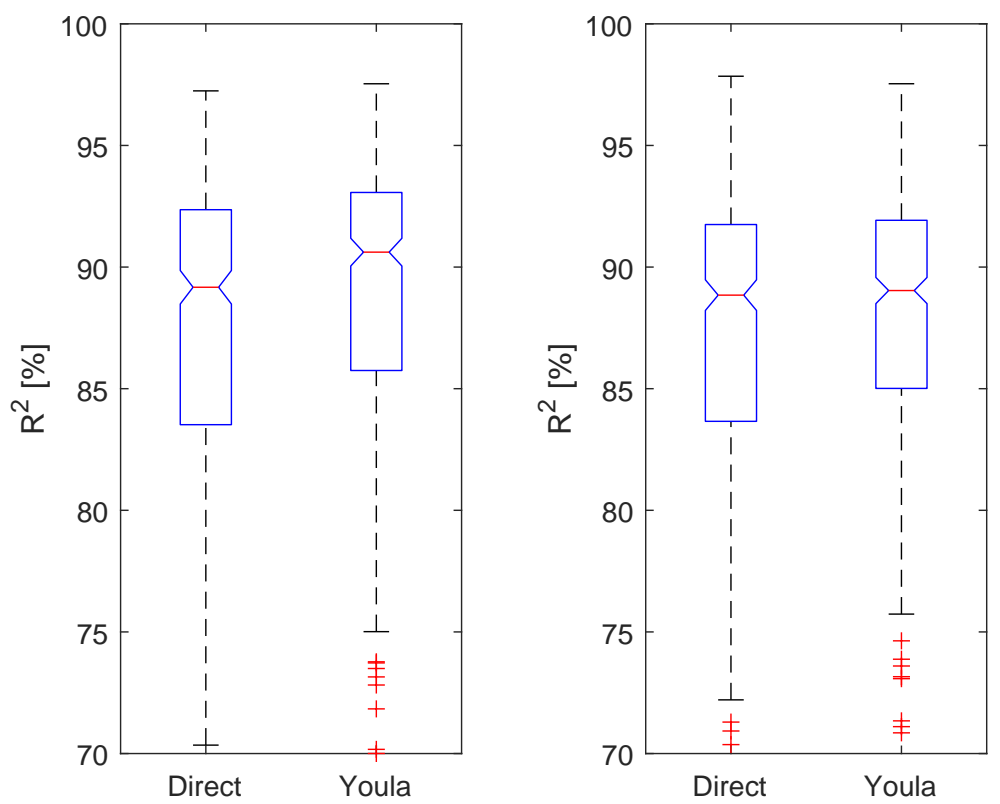

Figure 5. Boxplot of the $R^{2}$ fit given direct identification of the plant and identification of the Youla deviation system. The left plot is for the horizontal direction where as the right plot is for the vertical direction.

PEM is unable to identify a model of the same quality and obtains a lower $R^{2}$ fit when the nominal model is of such low quality. The same trend was observed in section when $\theta$ is equal to 0.5 . The result is summarised as column 2 in Table 1.

To further examine the effect of the identification methods for low quality nominal models, an experiment is conducted where the disc is rotating with 2500 revolutions per minute. Here it has instead been chosen to multiply all elements of the input matrix with 0.7 so that the input gain is higher than expected. The verification data together with the predicted output for each of the models are shown in Fig. 7.

The nominal model is better than in the previous example at predicting the position of the disc, however the nominal model is still having a fit lower than what is expected possible to obtain. This is seen from the relatively large increase in the fit for each of the identification methods. The result is summarised as column 1 in Table 1. Lastly an experiment is conducted to examine the identification methods ability to recover small deviations. Again the nominal model has been tampered with to degrade its performance. The elements of the system matrix have been multiplied by 0.97 so that the dynamics of the real plant is slightly faster than for the nominal model. Identification of the Youla deviation system using Eq. (28) has thus been conducted, 

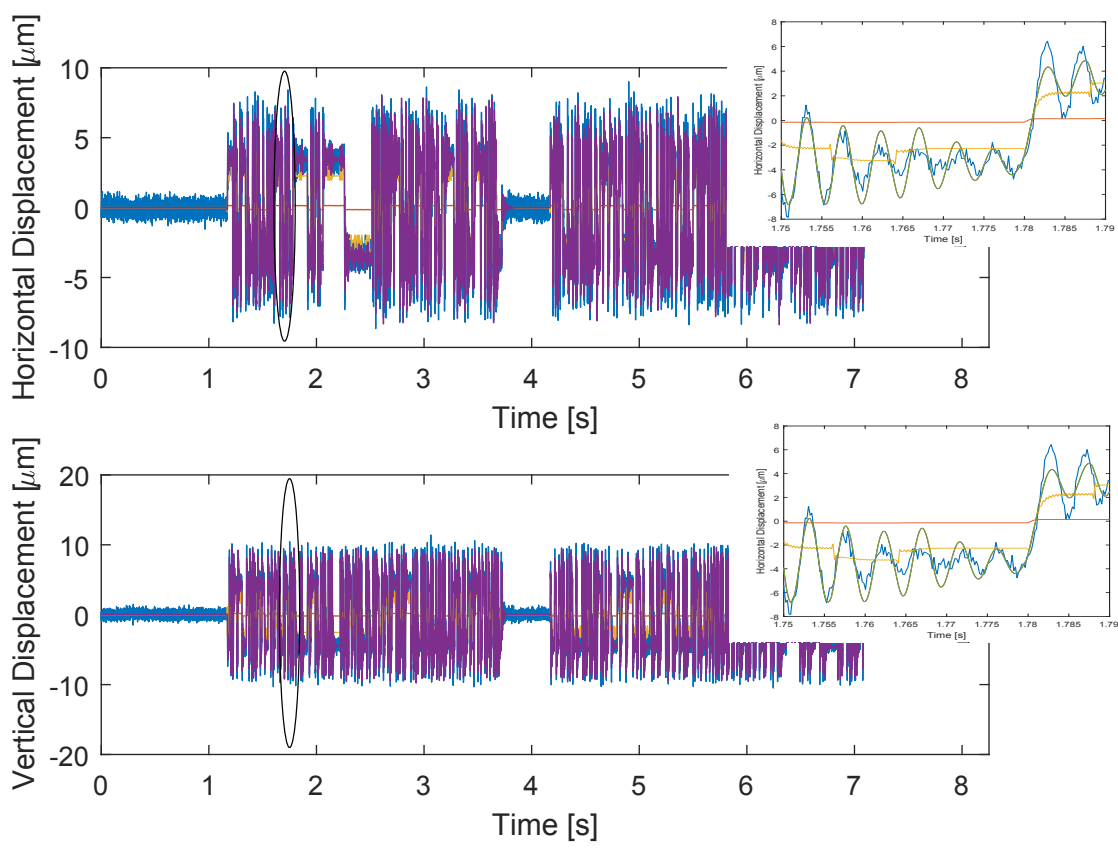

Figure 6. Comparison of a verification signal (blue) with the predicted output given the nominal model (red) and each of the 3 identified models. The nominal model $\mathrm{G}$ was found to have a fit of $3.188 \%$ in the horizontal direction and $2.999 \%$ in the vertical direction. The PEM (yellow) was found to have a fit of $48.91 \%$ in the horizontal direction and of 39.47 in the vertical direction. Identification of the Youla deviation system (purple) gave a fit of $76.76 \%$ in the horizontal direction and of $83.58 \%$ in the vertical direction.

and a plot of the signal $\beta$ measured and predicted using the identified system are shown in Fig. 8.

The nominal model and plant are fairly similar why noise is expected to dominated the $\beta$ signal. This is seen on Fig. 8 where the measured signal $\beta$ is presented. It is clear that the signal contain a dominant noise part in the horizontal direction. It is clear from Fig. 8 that the identification should improve the model in the vertical direction mainly. Again the identified model has been compared with a verification signal which is shown in Fig. 9.

It can be seen from Fig. 9 that the nominal model does indeed produce a better fit. Still, both methods are able to improve the model. As clearly seen from Fig. 8 the improvement of the identified model was mostly in the vertical direction. A zoom on the verification experiment is shown in Fig. 10.

It is clear from Fig. 10 that the identified models have improved the prediction accuracy in the vertical direction. Both models show a clear improvement compared to the nominal case as shown in the 3'rd column of Table 1 . The result of the 3 experiments 


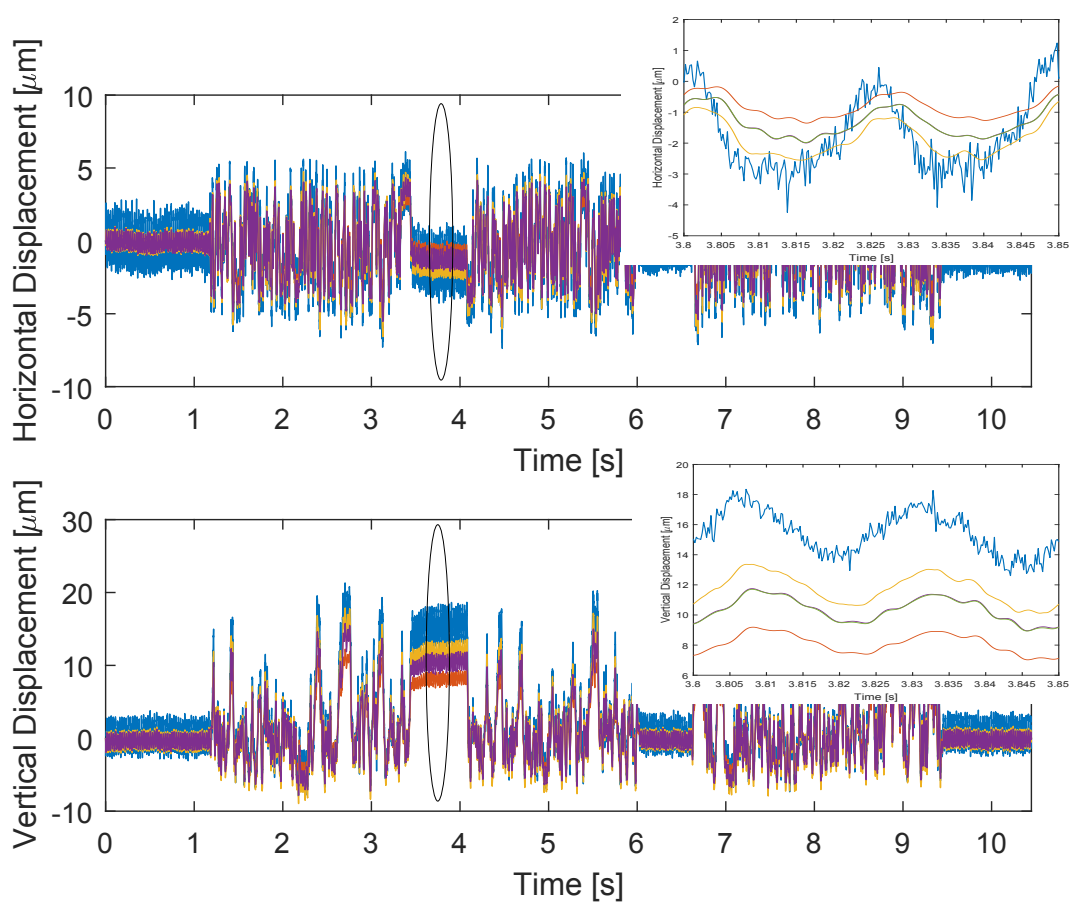

Figure 7. Comparison of a verification signal (blue) with the predicted output given the nominal model (red) and each of the 3 identified models. The nominal model $\mathrm{G}$ was found to have a fit of $51.36 \%$ in the horizontal direction and $54.13 \%$ in the vertical direction. The PEM (yellow) was found to have a fit of $62.87 \%$ in the horizontal direction and of 75.78 in the vertical direction. Identification of the Youla deviation system (purple) gave a fit of $61.04 \%$ in the horizontal direction and of $69.19 \%$ in the vertical direction.

for each of the identification methods using Eq. (26) to determine the quality of the identified model are shown in Table 1 together with the fit of the nominal model.

\begin{tabular}{|c|c|c|c|c|c|c|}
\hline & \multicolumn{2}{|c|}{2500 RPM } & \multicolumn{2}{|c|}{$\begin{array}{c}\text { high deviation } \\
\text { 0 RPM } \\
\end{array}$} & \multicolumn{2}{|c|}{$\begin{array}{c}\text { small deviation } \\
0 \mathrm{RPM}\end{array}$} \\
\hline & Horizontal & Vertical & Horizontal & Vertical & Horizontal & Vertical \\
\hline Nominal model & $51.36 \%$ & $54.13 \%$ & $3.19 \%$ & $3 \%$ & $83.21 \%$ & $76.12 \%$ \\
\hline Open loop PEM model & $62.87 \%$ & $75.78 \%$ & $48.91 \%$ & $39.47 \%$ & $83.9 \%$ & $84.63 \%$ \\
\hline Modified Hansen scheme $G(S)$ & $61.94 \%$ & $69.19 \%$ & $76.76 \%$ & $83.58 \%$ & $83.53 \%$ & $81.18 \%$ \\
\hline Modified Hansen scheme $G_{r e d}(S)$ & $60.71 \%$ & $69.33 \%$ & $76.73 \%$ & $83.53 \%$ & $83.07 \%$ & $79.59 \%$ \\
\hline
\end{tabular}

Table 1. Model fit using each of the two identification methods. The fit is $R^{2}$ fit shown in Eq. (26)

As seen in Table 1, using PEM or subspace identification of the Youla deviation system based on the modified Hansen scheme $(G(S))$ gives similar results for small deviations. 

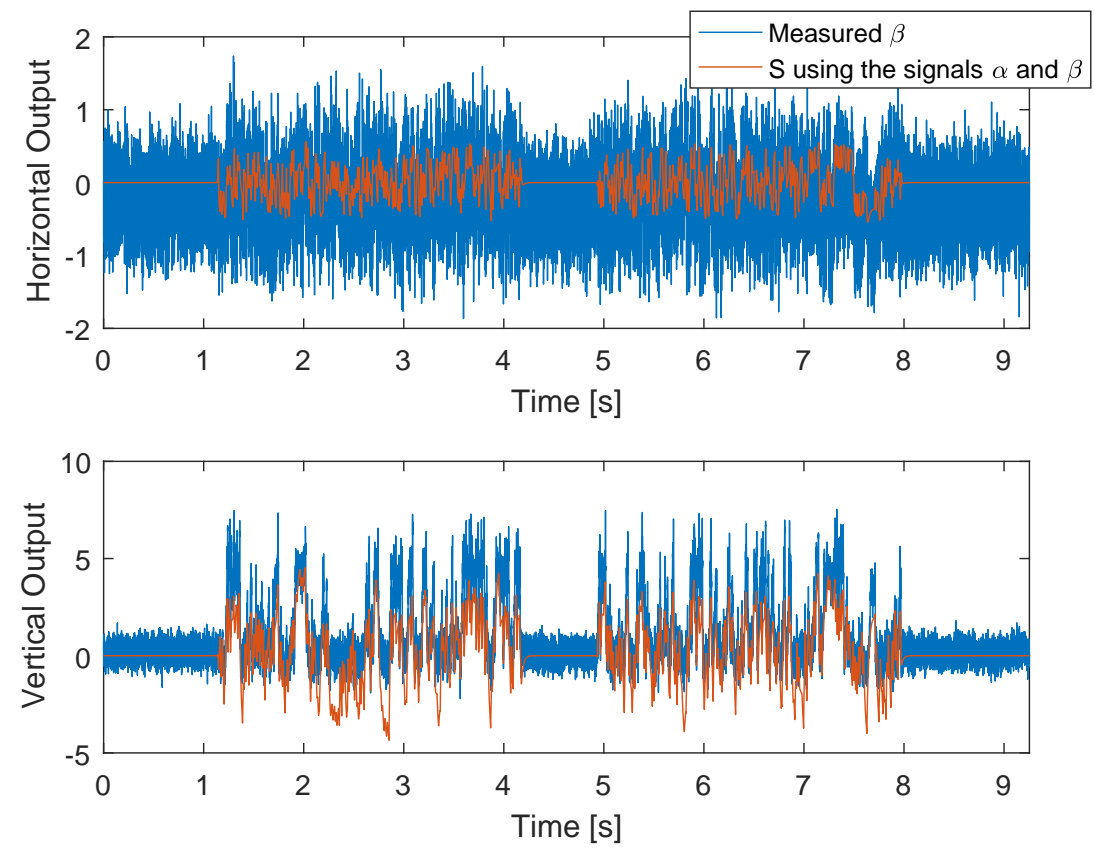

Figure 8. Comparison of the measured $\beta$ signal with the predicted $\beta$ signal, using the two identified models of the Youla deviation system. The blue line represents the measured signals, the red line is the predicted signals given an Youla deviation system identified using the modified Hansen Scheme.

However in the case where the nominal model lack significant insight to the plant(low fit for nominal model) the PEM results in a lower fit.

\section{Model reduction of the identified models}

The models identified using the modified Hansen scheme are all of a inconveniently high order. This is a well known result of the identification method and it is therefore found useful to investigate the impact on the quality of the identification when model reduction is conducted. Model reduction has in this paper been conducted by requiring all models to be of the same order as the nominal model ( $6^{\prime}$ th order). The same systems as identified previously have been compared in Table 1 where those systems identified using the Hansen scheme have been reduced to be of $6^{\prime}$ th order. The identified plant after model reduction is given in Eq. (29).

$$
G_{r e d}(S)=\operatorname{modred}(G(S))
$$



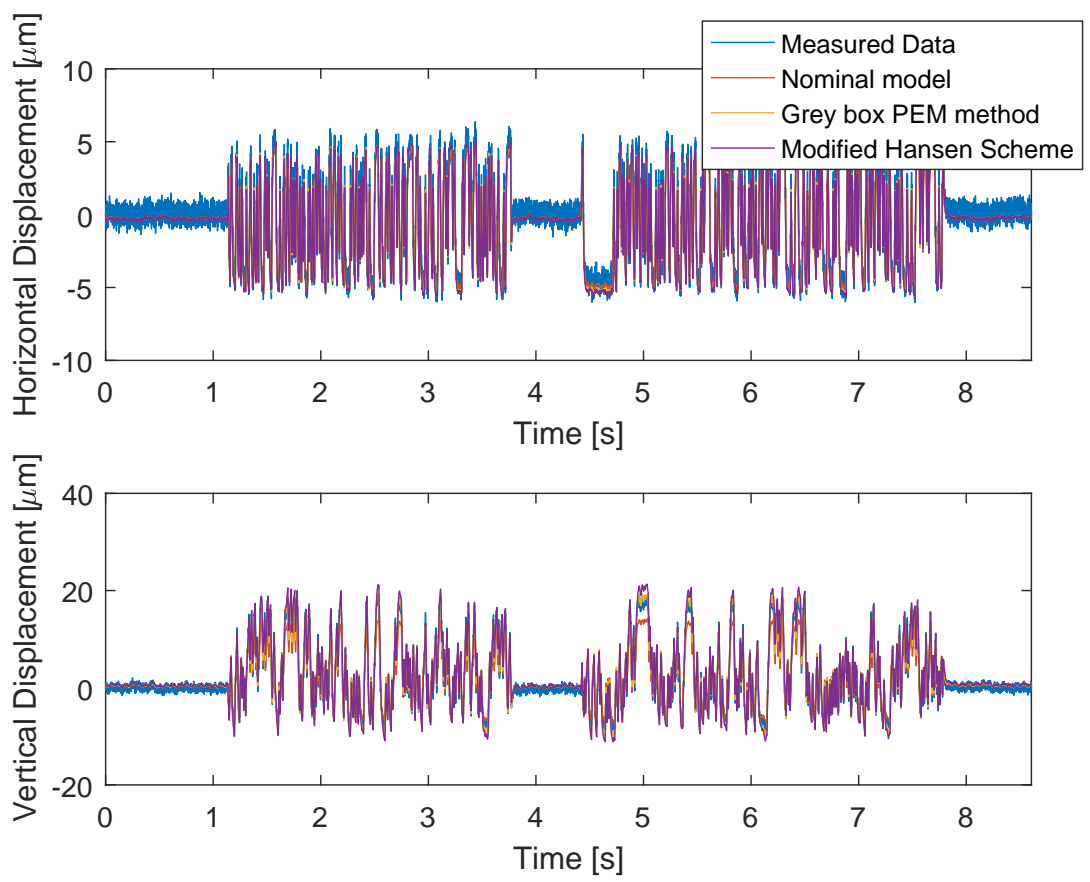

Figure 9. Comparison of a verification signal (blue) with the predicted output given the nominal model (red) and each of the 3 identified models. The nominal model $G$ was found to have a fit of $81.93 \%$ in the horizontal direction and $76.12 \%$ in the vertical direction. The PEM (yellow) was found to have a fit of $83.9 \%$ in the horizontal direction and of 84.63 in the vertical direction. Identification of the Youla deviation system (purple) gave a fit of $83.53 \%$ in the horizontal direction and of $81.18 \%$ in the vertical direction.

Here $\operatorname{modred}()$ denotes the model reduction function used and $G(S)$ is the originally identifed plant using the modified Hansen scheme. The results of the models for which model reduction has been conducted are shown in Table 1 . The model reduction technique used is described in ${ }^{18}$ and the resulting $6^{\prime}$ th order approximations shows to have a similar fit to the full order identified systems as shown in Tab. 1. It is worth noting that the initially identified models had as much as 40 states, which makes control design problematic.

\section{Conclusion}

Identification of a gas bearing as part of a closed loop system has been conducted. It was shown possible to identify the gas bearing, as part of a closed loop system, by reformulating the problem into identification of the Youla deviation system which is a standard open loop identification problem. Furthermore, using an open loop PEM method for identification gives similar results when the nominal model predicts the system dynamics with high accuracy. A simulation example was given which pointed 

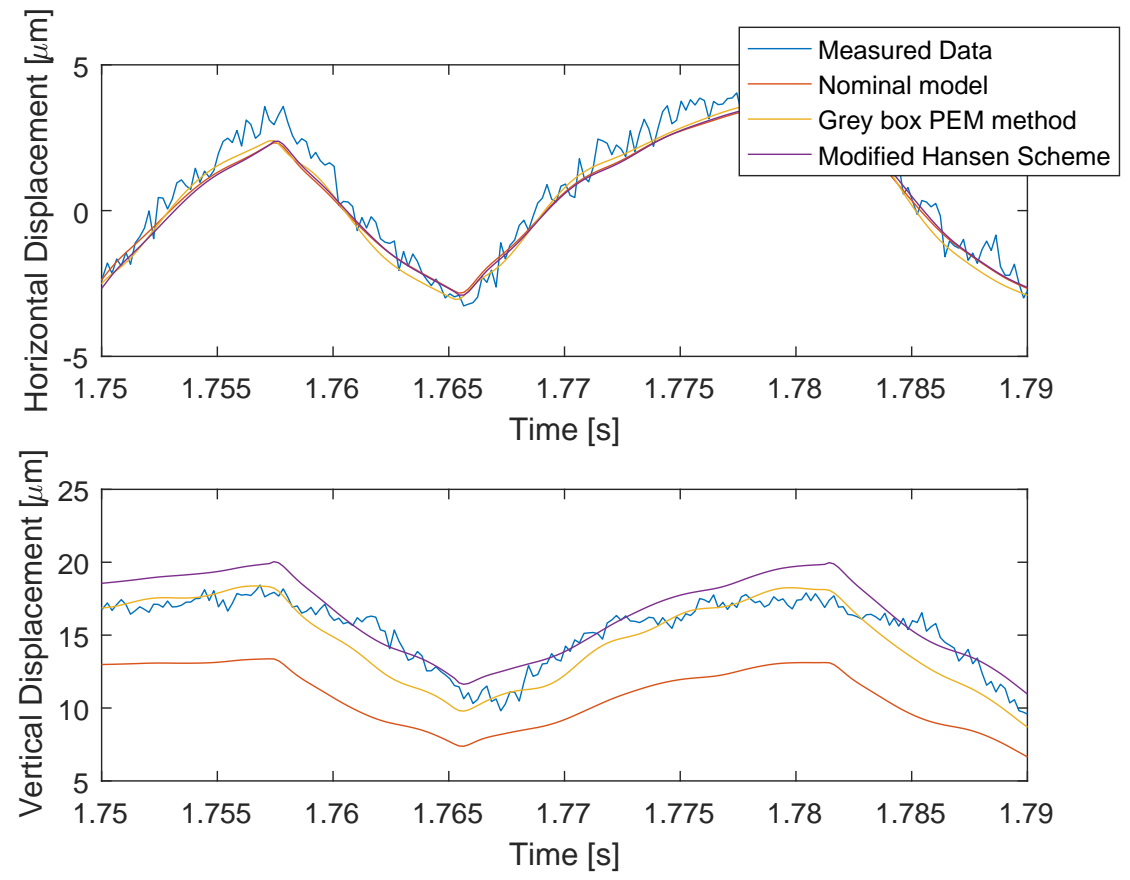

Figure 10. Zoom in on the comparison of a verification signal(blue) with the predicted output given the nominal model (red) and each of the 2 identified models, PEM (yellow) and Youla deviation system (purple)

towards the identification of the youla deviation system to produce better results on average for poor knowledge of the plant dynamics. Experiments showed that for a poor nominal model the modified Hansen scheme method did indeed produce better results, with a fit $28 \%$ in the horizontal direction and $44 \%$ higher in the vertical direction for poor a priori knowledge of the plant dynamics. The PEM method furthermore used considerably longer time to identify a model than the subspace identification method used to determine the Youla deviation system. This result might be due to how the PEM method is restricted to the model structure determined while the modified Hansen scheme is not. The identified systems using the Youla deviation system however also has some disadvantages. Any physical understanding related to model parameters of the gas bearing is lost when using the modified Hansen scheme. Furthermore the methods produced models of higher order than the nominal model and the plant identified using the PEM for identification. This tendency of order increase comes naturally from the construction of the plant when the Youla deviation system has been identified. Unnecessary high order model solutions is thus a well known consequence when using the modified Hansen scheme. Model reduction has therefore been conducted which was able to produce acceptable results. It have thus been shown that the method has its clear advantage over PEM identification when model expert knowledge is lacking. 


\section{Conflict of interest}

The authors declare no conflict of interest in preparing this article.

\section{Funding}

This research received no specific grant from any funding agency in the public, commercial, or not-for-profit sectors.

\section{References}

1. F. G. Pierart and I. F. Santos, "Steady state characteristics of an adjustable hybrid gas bearing-computational fluid dynamics, modified reynolds equation and experimental validation," Proceedings of the Institution of Mechanical Engineers, Part J: Journal of Engineering Tribology, vol. 229, no. 7, pp. 807-822, 2015.

2. — , "Active lubrication applied to radial gas journal bearings. part 2: Modelling improvement and experimental validation," Tribology International, vol. 96, pp. 237-246, 2016.

3. — - "Adjustable hybrid gas bearing-influence of piezoelectrically adjusted injection on damping factors and natural frequencies of a flexible rotor operating under critical speeds," Proceedings of the Institution of Mechanical Engineers, Part J: Journal of Engineering Tribology, vol. 230, no. 10, pp. 1209-1220, 2016.

4. _ _ "Lateral vibration control of a flexible overcritical rotor via an active gas bearingtheoretical and experimental comparisons," Journal of Sound and Vibration, vol. 383, pp. 20-34, 2016.

5. L. R. Theisen, H. H. Niemann, R. Galeazzi, and I. F. Santos, "Enhancing damping of gas bearings using linear parameter-varying control," Journal of Sound and Vibration, vol. 395, pp. 48-64, 2017.

6. L. R. S. Theisen and H. Niemann, "Modelling of rotor-gas bearings for feedback controller design," in Journal of Physics: Conference Series, vol. 570. IOP Publishing, 2014, p. 052005.

7. L. R. S. Theisen, F. G. Pierart, H. Niemann, I. F. Santos, and M. Blanke, "Experimental grey box model identification and control of an active gas bearing," in Vibration Engineering and Technology of Machinery. Springer, 2015, pp. 963-976.

8. S. J. Qin, "An overview of subspace identification," Computers \& chemical engineering, vol. 30, no. 10, pp. 1502-1513, 2006.

9. P. Van Overschee and B. De Moor, "N4SID: Subspace algorithms for the identification of combined deterministic-stochastic systems," Automatica, vol. 30, no. 1, pp. 75-93, 1994.

10. M. Verhaegen and P. Dewilde, "Subspace model identification part 1. the output-error statespace model identification class of algorithms," International journal of control, vol. 56, no. 5, pp. 1187-1210, 1992.

11. F. Hansen, G. Franklin, and R. Kosut, "Closed-loop identification via the fractional representation: Experiment design," in 1989 American Control Conference, 1989, pp. 14221427.

12. A. Sekunda, H. Niemann, N. K. Poulsen, and I. Santos, "Closed loop identification using a modified hansen scheme," Journal of Physics: Conference Series, vol. 659, no. 1, p. 012009, 2015. [Online]. Available: http://stacks.iop.org/1742-6596/659/i=1/a=012009

Prepared using sagej.cls 
13. S. Morosi and I. F. Santos, "Active lubrication applied to radial gas journal bearings. part 1: Modeling,” Tribology International, vol. 44, no. 12, pp. 1949-1958, 2011.

14. F. G. P. Vásquez, "Model-based control design for flexible rotors supported by active gas bearings-theory \& experiment," PhD thesis, 2016.

15. K. Zhou and J. C. Doyle, Essentials of robust control. Prentice hall Upper Saddle River, NJ, 1998.

16. B. D. Anderson, "From Youla-Kucera to identification, adaptive and nonlinear control," Automatica, vol. 34, pp. 1485-1506, 1998.

17. L. Ljung, "System identification," in Signal Analysis and Prediction. Springer, 1998, pp. $163-173$.

18. A. Varga, "Balancing free square-root algorithm for computing singular perturbation approximations," in Decision and Control, 1991., Proceedings of the 30th IEEE Conference on. IEEE, 1991, pp. 1062-1065.

Prepared using sagej.cls 